Research Paper

\title{
KPNA2 promotes migration and invasion in epithelial ovarian cancer cells by inducing epithelial-mesenchymal transition via Akt/GSK-3ß/Snail activation
}

Long Huang 1, 2, 3, Yun Zhou 1, 4, 5, Xin-Ping Cao 4, 5, 6, Jia-Xin Lin7, Lan Zhang1, 4, 5, Shu-Ting Huang1, 4, 5, Min Zheng1,4,5凶

${ }^{1}$ Department of Gynecology, Sun Yat-Sen University Cancer Center, Guangzhou, China; 2 Department of Oncology, The Second Afiliated Hospital of Nanchang University, Nanchang, China; ${ }^{3}$ JiangXi Key Laboratory of Clinical and Translational Cancer Research; ${ }^{4}$ State Key Laboratory of Oncology in South China; 5

Collaborative Innovation Center for Cancer Medicine, Guangzhou, China; ${ }^{6}$ Department of Radiation, Sun Yat-Sen University Cancer Center, Guangzhou, China;

7 Department of Oncology, Guangdong General Hospital, Guangzhou, China.

$\triangle$ Corresponding author: Min Zheng, Department of Gynecology, Sun Yat-sen University Cancer Center, State Key Laboratory of Oncology in South China, Collaborative Innovation Center for Cancer Medicine, 651 Dongfeng Road East, Guangzhou 510060, P.R. China. Phone: 86-20-32968086; Fax: 86-20-32968086; E-mail: zhengmin@sysucc.org.cn

(c) Ivyspring International Publisher. This is an open access article distributed under the terms of the Creative Commons Attribution (CC BY-NC) license (https://creativecommons.org/licenses/by-nc/4.0/). See http://ivyspring.com/terms for full terms and conditions.

Received: 2017.05.05; Accepted: 2017.10.06; Published: 2018.01.01

\begin{abstract}
Background: Increased karyopherin alpha 2 (KPNA2) expression has been demonstrated in epithelial ovarian carcinoma (EOC) tissue. However, its role in the disease is not clear. Here, we investigate the mechanism of involvement of KPNA2 in EOC.

Methods: Stable cell lines expressing KPNA2, or KPNA2 shRNAs, were constructed. The effects of KPNA2 overexpression and knockdown on EOC cell migration, invasion, and epithelial-to-mesenchymal transition (EMT) were evaluated using relevant assays and western blot analysis. Key components of the Akt/GSK-3ß/Snail signaling pathway were detected using western blotting and immunofluorescence.

Results: KPNA2 overexpression increased the migration and invasion of EOC cells (EFO-21 and SK-OV3); these cells also exhibited characteristics of EMT. Key proteins in the Akt/GSK-3ß/Snail signaling pathway were also upregulated in cells overexpressing KPNA2. In contrast, knockdown of KPNA2 effectively suppressed migration and invasion of these EOC cells.

Conclusions: KPNA2 may reduce the migration and invasion of EOC by inhibiting the Akt/GSK-3 $\beta /$ Snail signaling pathway and suppressing EMT.
\end{abstract}

Key words: KPNA2, EOC

\section{Introduction}

Epithelial ovarian carcinoma (EOC) kills more women than any other gynecological malignancy. In 2014 , it is estimated there were $>14,000$ EOC deaths and nearly 22,000 new cases [1]. The condition is often diagnosed late (at an advanced stage) and has a high metastasis rate. It is therefore crucial to understand the molecular mechanisms of EOC and to improve the available therapies.

Deregulation of cellular transport machinery occurs frequently in tumors. Increased expression of karyopherin alpha 2 (KPNA2), a protein that functions in nucleocytoplasmic transport, has been shown in melanoma [2], and cancers of the cervix [3], esophagus [4], lung [5], prostate [6], brain [7], liver [8], and bladder [9]. KPNA2 is overexpressed in human EOC tissues and cell lines [10], and overexpression is correlated with poor prognosis in EOC [10]. Nevertheless, the mechanism of KPNA2 in EOC pathogenesis remains to be conclusively determined. Here, we investigated the effects of KPNA2 
overexpression and knockdown in EOC cell lines in vitro.

\section{Materials and Methods}

\section{Cell culture}

Ovarian cancer cell lines SKOV3 and EFO21 were grown in Dulbecco's Modified Eagle's Medium (Invitrogen, Carlsbad, CA, USA) containing 10\% fetal bovine serum (FBS; HyClone, Logan, UT, USA) and $1 \%$ penicillin-streptomycin. Cells were cultured at $37^{\circ} \mathrm{C}$ under a humidified atmosphere containing $5 \%$ $\mathrm{CO}_{2}$.

To overexpress KPNA2, a full-length human KPNA2 cDNA was ligated into plasmid pMSCV. To knockdown KPNA2, short hairpin (sh) RNA sequences were cloned into pSuper-retro-puro (Langri, GuangZhou, China) to generate RNAis with sequences RNAi\#1, 5'-ATTTACAGTGCCCTGGTTG$3^{\prime}$ and RNAi\#2, 5'-TTAACGAAGCC TTATACAC-3'. Transfection of plasmids used Lipofectamine 2000 reagent (Invitrogen) according to the manufacturer's protocol. Cell lines stably-expressing KPNA2 or the KPNA2 shRNAs were treated with $0.5 \mathrm{mg} / \mathrm{mL}$ puromycin for 10 days.

\section{Western blot assays}

Western blot analysis was performed according to standard methods, as previously described [10], using anti-KPNA2 (Abcam, Cambridge, UK), anti-total Akt, anti-p-Akt, anti-total GSK-3 $\beta$, anti-p-GSK-3 $\beta$, anti-snail, anti-twist, anti-vimentin, anti-E-cadherin, anti-Fibronectin, and anti-a-catenin antibodies (Cell Signaling Technology, Danvers, MA, USA). Polyvinylidene fluoride membranes were stripped and re-blotted with an anti- $\beta$-actin monoclonal antibody (Sigma, St. Louis, MO, USA) as a loading control.

\section{Wound-healing assay}

Cells $\left(2 \times 10^{6}\right)$ were seeded into $35-\mathrm{mm}$ Petri dishes. After 12-h of incubation, a wound was created using a P200 pipette tip. Microscopic images were recorded immediately $(0 \mathrm{~h})$ and after 8 and $16 \mathrm{~h}$.

\section{Invasion assays}

Cell invasiveness used Matrigel-coated Invasion Chambers (8 $\mu \mathrm{m}$; BD Biosciences, San Jose, CA) according to the manufacturer's protocol. Transfected cells $(\sim 25,000$ per well) were resuspended in serum-free medium, then transferred to the upper chamber of a Matrigel-coated insert. Medium in the lower chamber included 10\% FBS. Cells were incubated at $37^{\circ} \mathrm{C}$ for $24 \mathrm{~h}$, then cells on the upper surface were removed and discarded. Cells that had invaded the lower surface were fixed and stained with $0.05 \%$ crystal violet for $30 \mathrm{~min}$ and counted microscopically.

\section{Immunofluorescence analysis}

Cells were incubated briefly with primary antibodies against a-catenin, vimentin, E-cadherin or $\mathrm{N}$-cadherin, and then incubated with rhodamine-conjugated or FITC-conjugated goat anti-rabbit or anti-mouse IgG. Coverslips were counterstained with 4',6-diamidino-2-phenylindole. Images were collected using a confocal laser-scanning microscope (FV1000, Olympus) and processed in Adobe Photoshop 7.0.

\section{Statistical analysis}

Statistical analysis was performed using the SPSS software package (version 16.0, SPSS). A $P$-value of $<0.05$ was considered statistically significant. For the in vitro studies, statistical significance was verified using a Student's t-test (two tailed).

\section{Results}

\section{KPNA2 is essential for EOC cell migration and invasion}

EFO-21 and SK-OV3 cell lines were used to stably-express KPNA2 cDNA (i.e., EFO-21/KPNA2 and SK-OV3/KPNA2) and KPNA2 shRNAs (i.e., EFO-21/siKPNA2\#1, EFO-21/siKPNA2\#2, SK-OV3/siKPNA2\#1, and SK-OV3/siKPNA2\#2). In wound-healing assays, upregulation of KPNA2 expression significantly increased migration and invasion of EOC cells, while downregulation of KPNA2 expression significantly reduced migration and invasion of EOC cells $(P<0.05 ;$ Fig. 1). Moreover, the invasiveness of EOC cells were dramatically expedited by the upregulation of KPNA2 expression, and impeded by the downregulation of KPNA2 expression, as shown in the invasion assays (Fig. 2).

\section{KPNA2 promotes epithelial-to-mesenchymal transition (EMT) and activates the Akt/GSK-3 $\beta /$ Snail pathway}

Overexpression of KPNA2 augmented the expression of mesenchymal markers such as vimentin, fibronectin, and $\mathrm{N}$-cadherin but inhibited the expression of epithelial markers (including $\alpha$-catenin), as well as E-cadherin (Fig. 3A-D, Fig. 4). Alternatively, knockdown of KPNA2 suppressed the expression of mesenchymal markers but enhanced the expression of epithelial markers (Figs. 3A and 3E-K, Fig. 4). Upregulating KPNA2 also increased the expression of phosphorylated Akt, but not total Akt; the expression of Snail, Twist, and phosphorylated GSK-3 $\beta$ were also upregulated, but not total GSK-3 $\beta$ 
(Fig. 3A-D, Fig. 4). Meanwhile, silencing KPNA2 repressed the expression of Snail, Twist, and
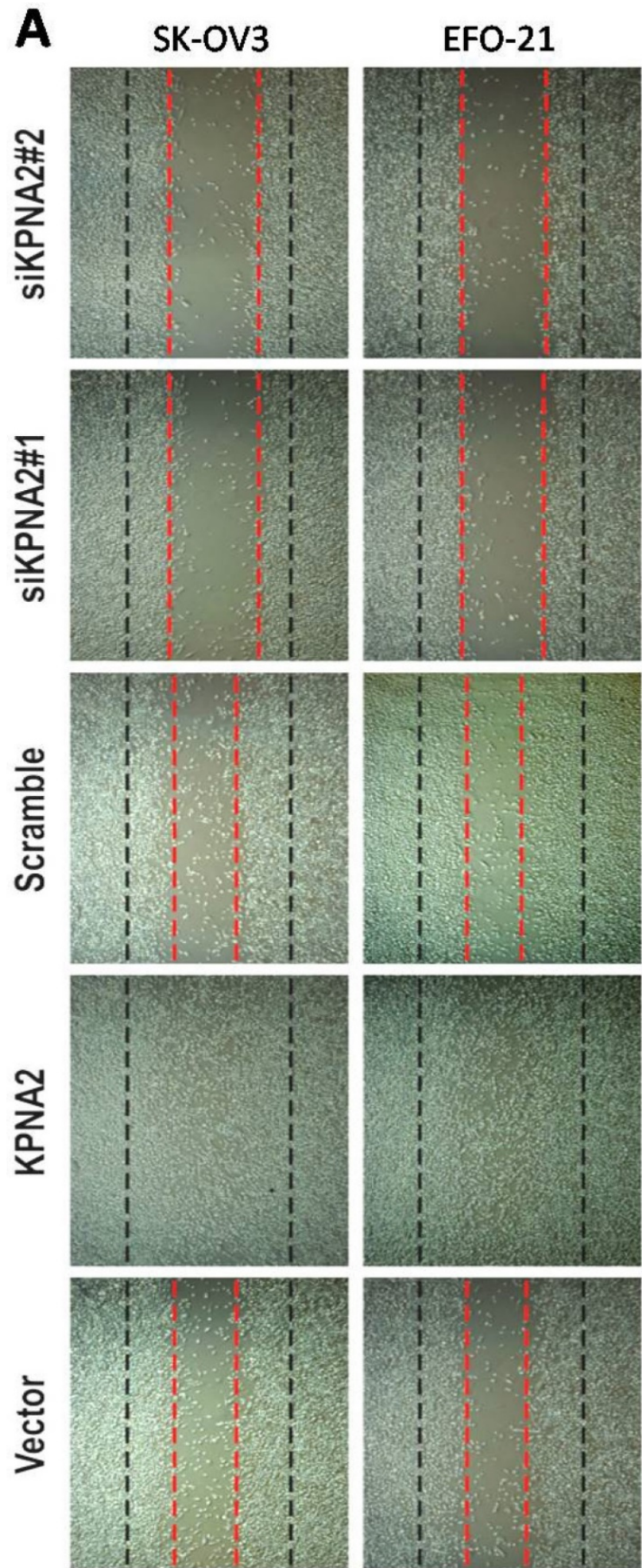

Figure 1. Effects of KPNA2 on epithelial ovarian carcinoma (EOC) cell motility. A, Motility as measured by testing the wound closure rate at 0 hours (red dotted line) and 36 hours (black dotted line) $(\times 200$; $* P<0.001)$. B, Representative micrographs, and quantification of wound closure rate in EFO-21 and SK-OV 3 cell lines stably overexpressing KPNA2 or infected with KPNA2 shRNA(s) relative to the control. Data were obtained from three independent experiments and described similar results. phosphorylated Akt/GSK-3 $\beta$ (Figs. $3 \mathrm{~A}$ and 3E-K, Fig. 4).

\section{B}
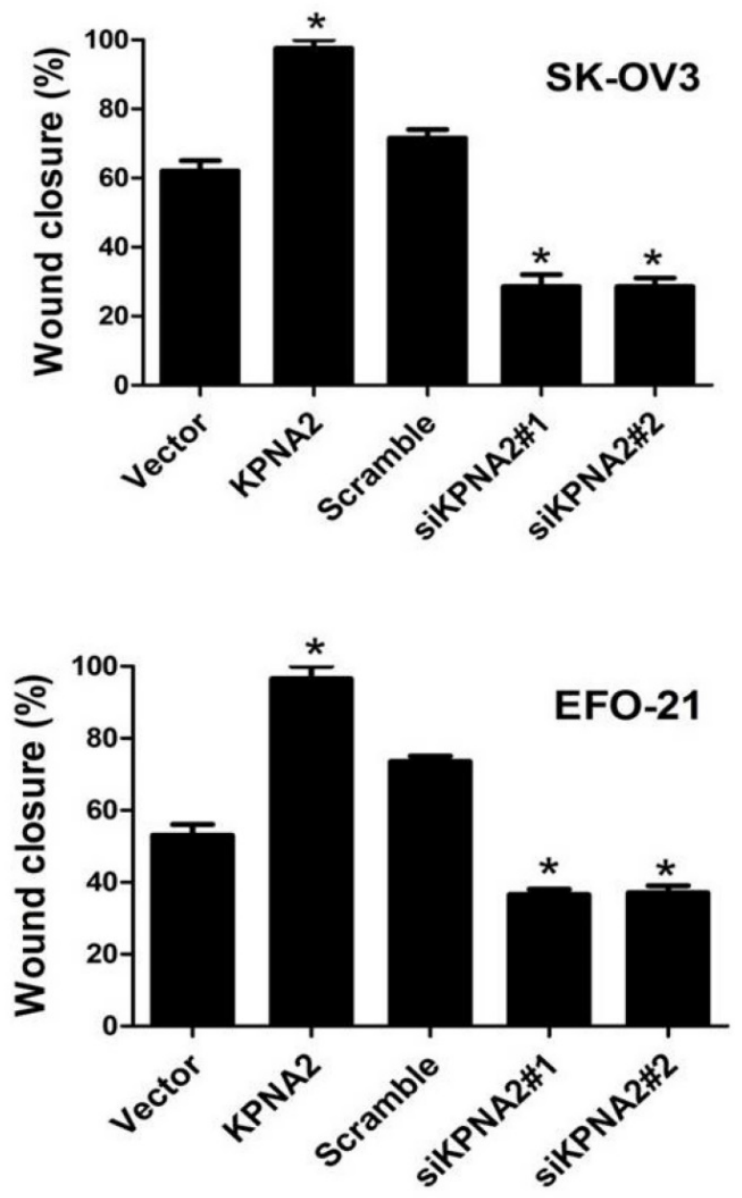
A

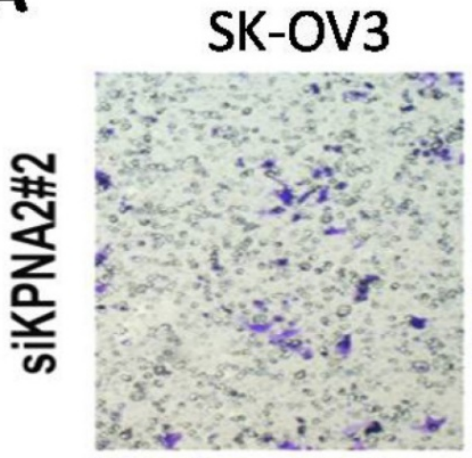

EFO-21

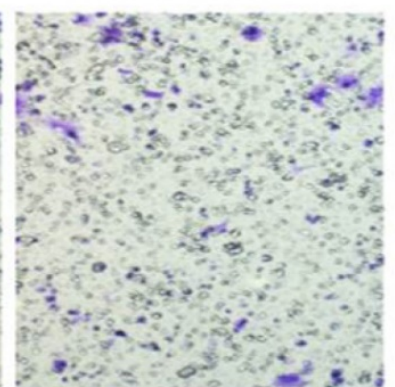

B
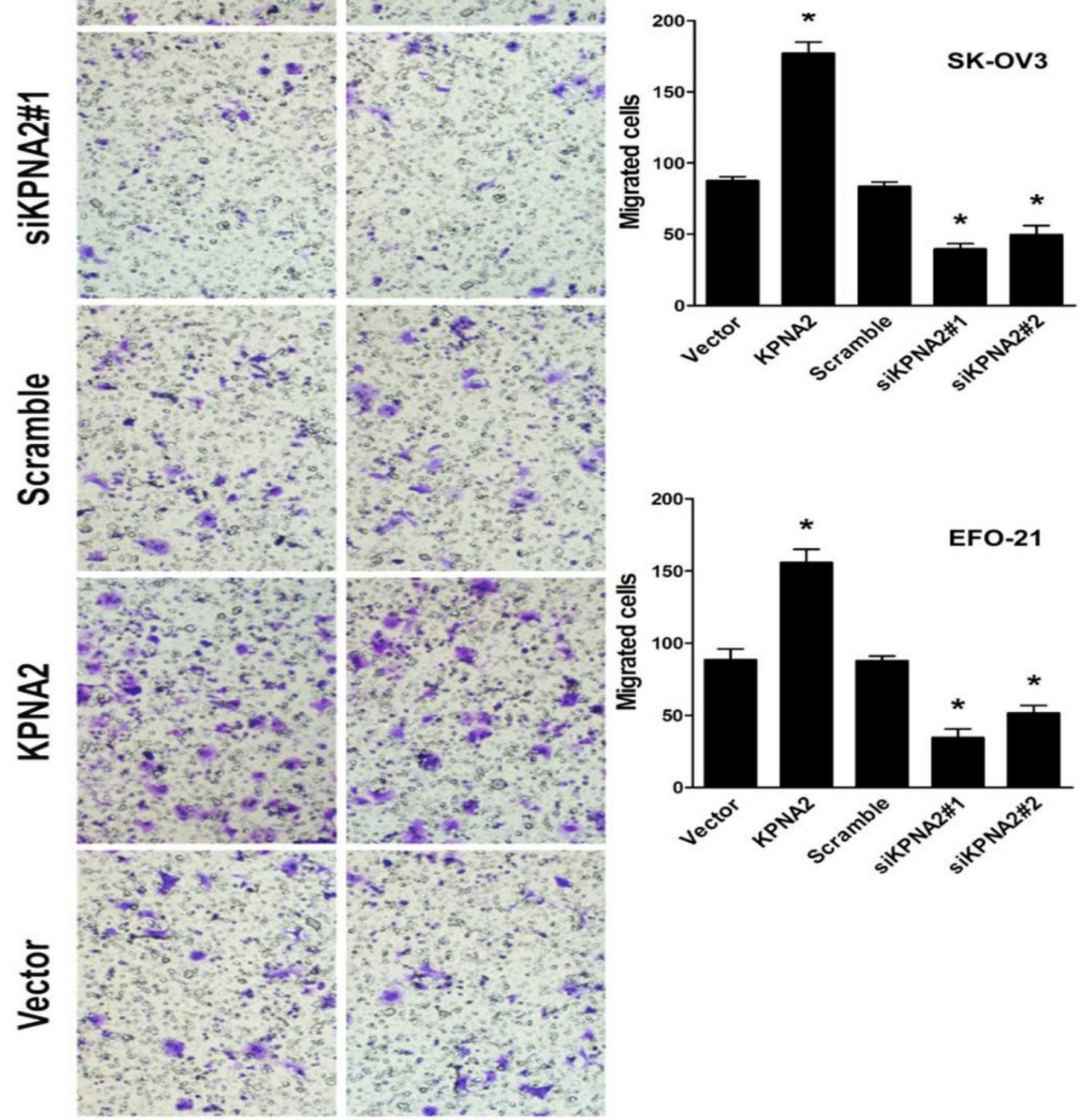

Figure 2. Effect of KPNA2 on EOC cell invasion. A, Invasion ability induced by fetal bovine serum as analyzed by the Transwell migration assay ( $\times 200$; $* P<0.001)$. B, quantification of invasive properties induced by fetal bovine serum in EFO-21 and SK-OV3 cell lines stably overexpressing KPNA2 or infected with KPNA2 shRNA(s) relative to the control. Data were obtained from three independent experiments and described similar results. 
A
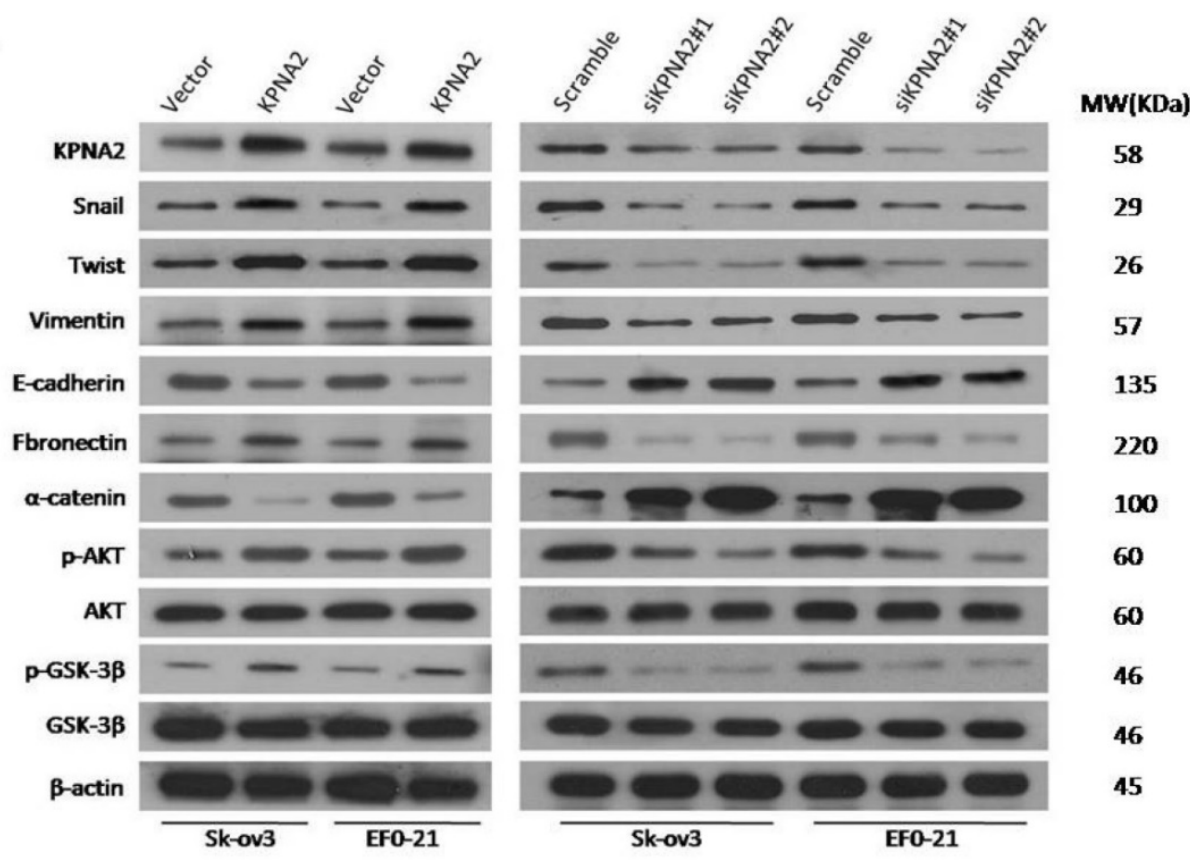

B

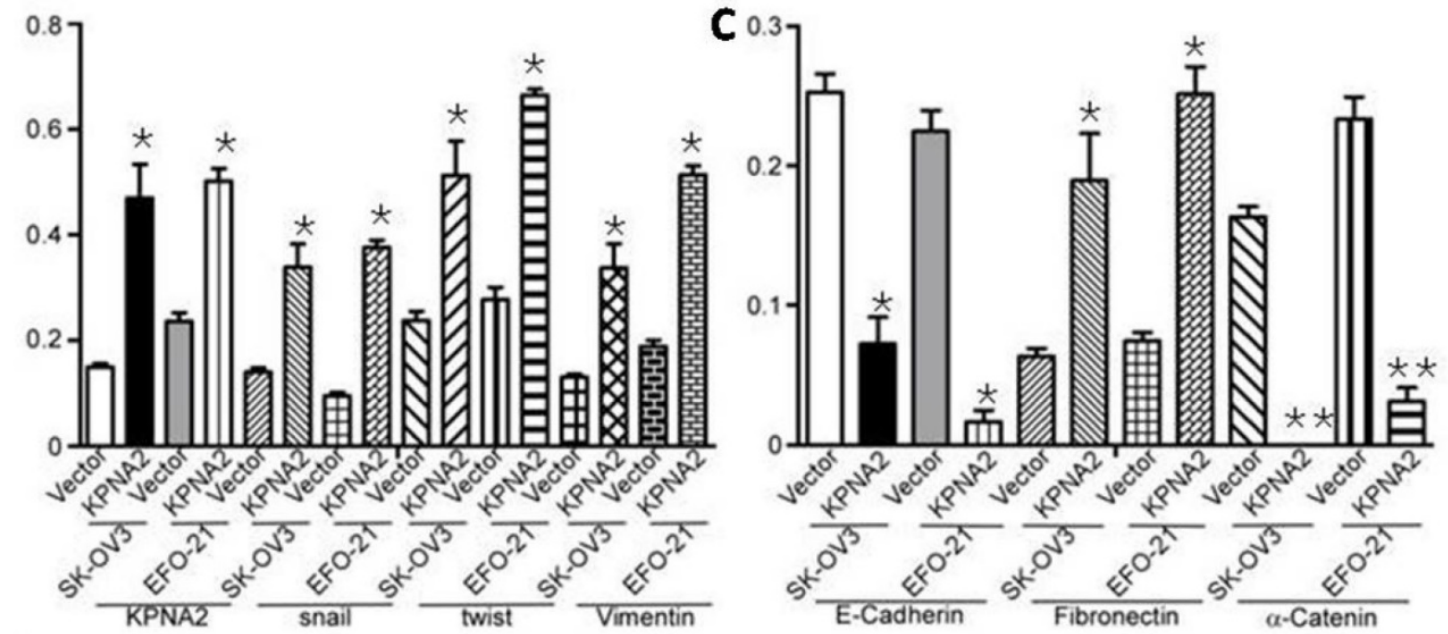

D
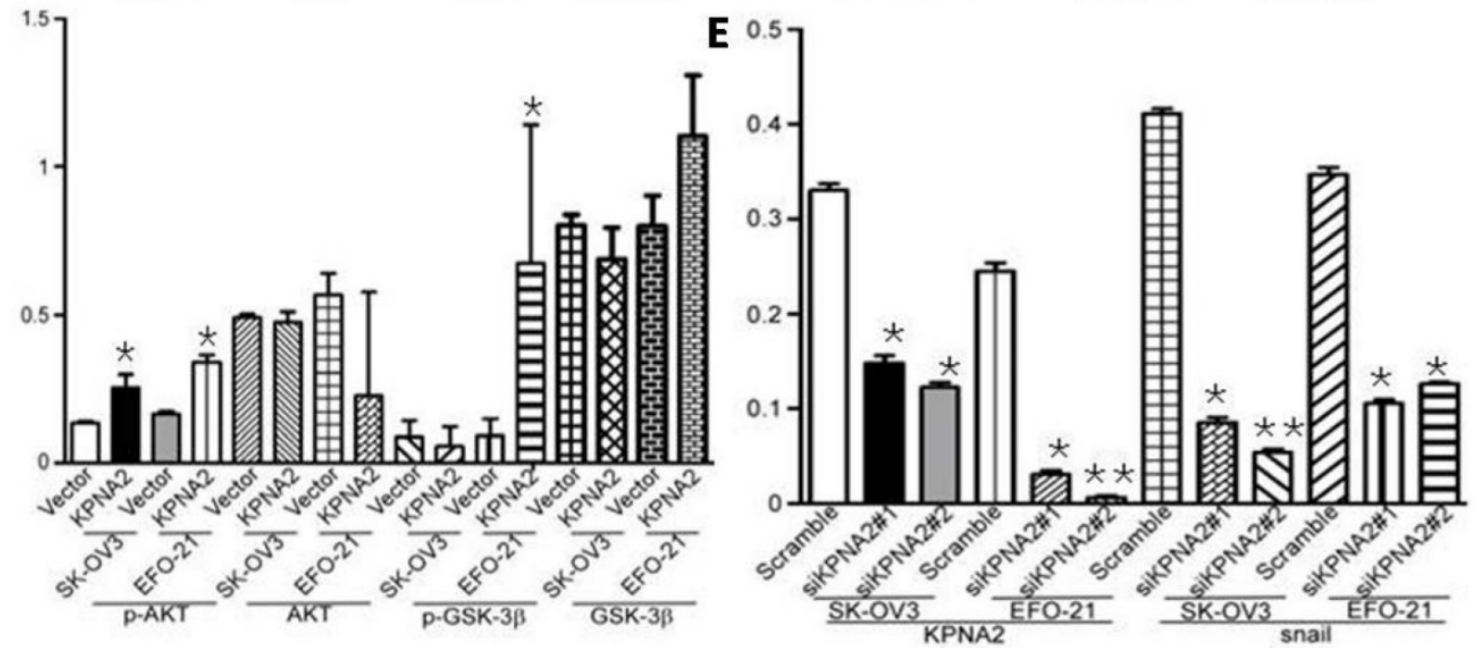

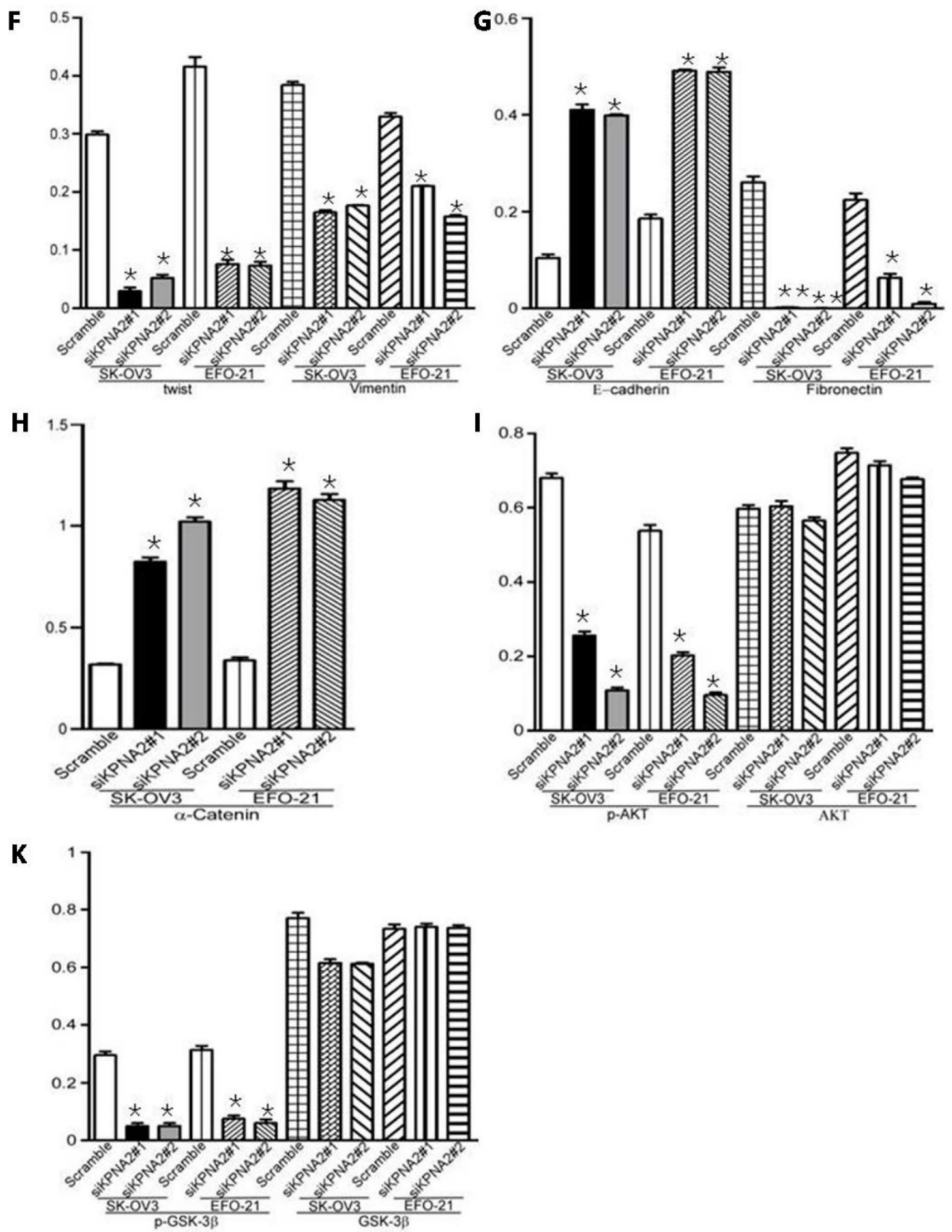

Figure 3. Effect of KPNA2 on epithelial-to-mesenchymal transition (EMT) and the Akt/GSK-3ß/Snail pathway. A, Western blot analysis of KPNA2, Akt, p-Akt,

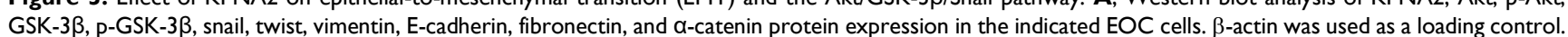
B-K, Expression levels were quantified using Image] software. Error bars represent the standard deviation (SD) of three independent experiments. Expression levels were normalized to $\beta$-actin $(* P<0.001, * * P<0.0001)$. 

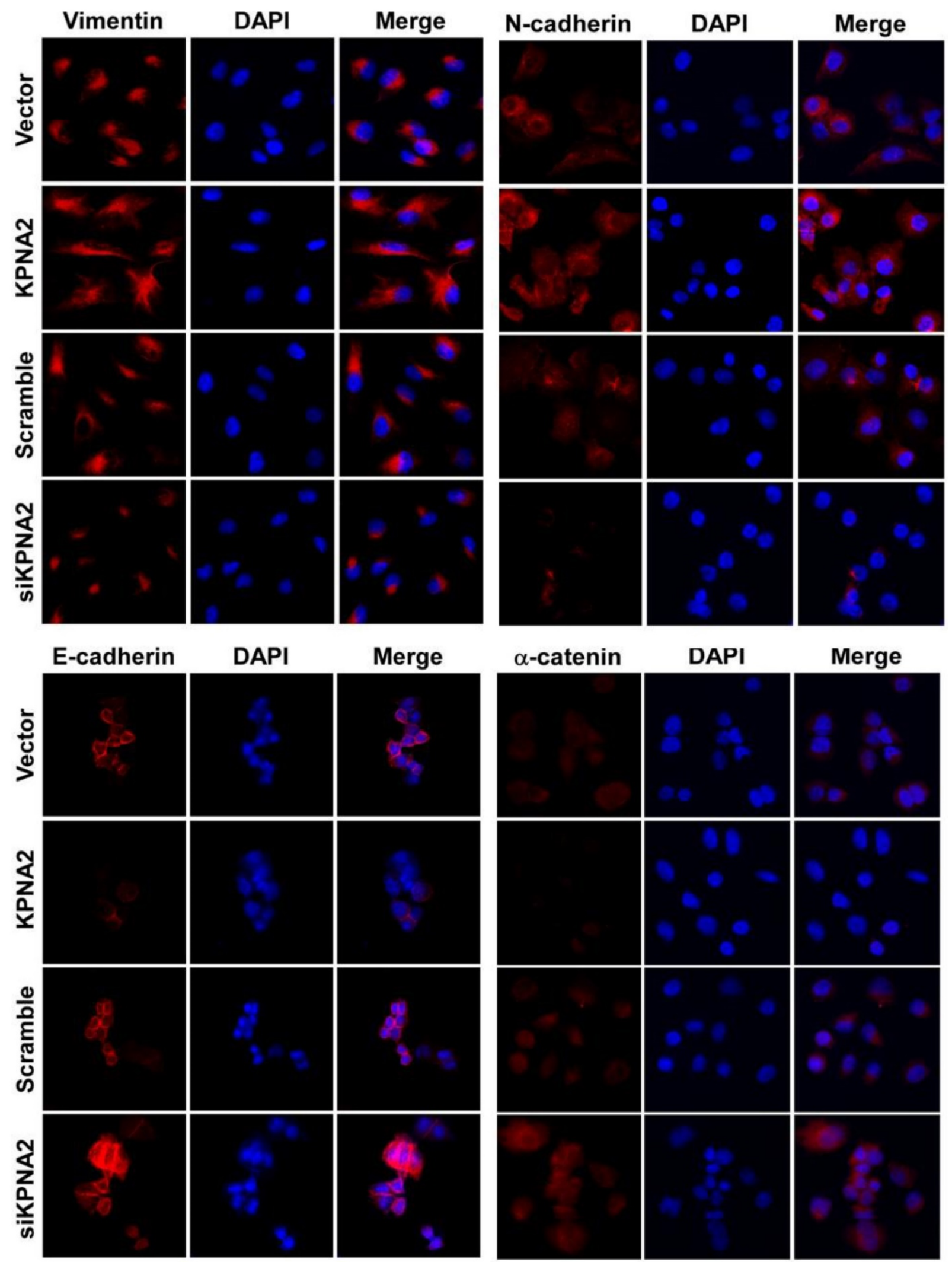

Figure 4. Effect of KPNA2 gene expression on epithelial-to-mesenchymal transition in EOC cells. KPNA2-overexpressing and KPNA2 shRNA(s)-infected SK-OV3 cells were placed on coverslips pre-coated with 10\% fetal bovine serum/RPMI-1640. After an additional 24 hours, cells were stained for E-cadherin, $\alpha$-catenin, $\mathrm{N}$-cadherin, vimentin, and 4',6-diamidino-2-phenylindole (DAPI) and analyzed by confocal microscopy. The red signal represents staining for the corresponding protein, while the blue signal signifies nuclear DNA staining with rhodamine. 


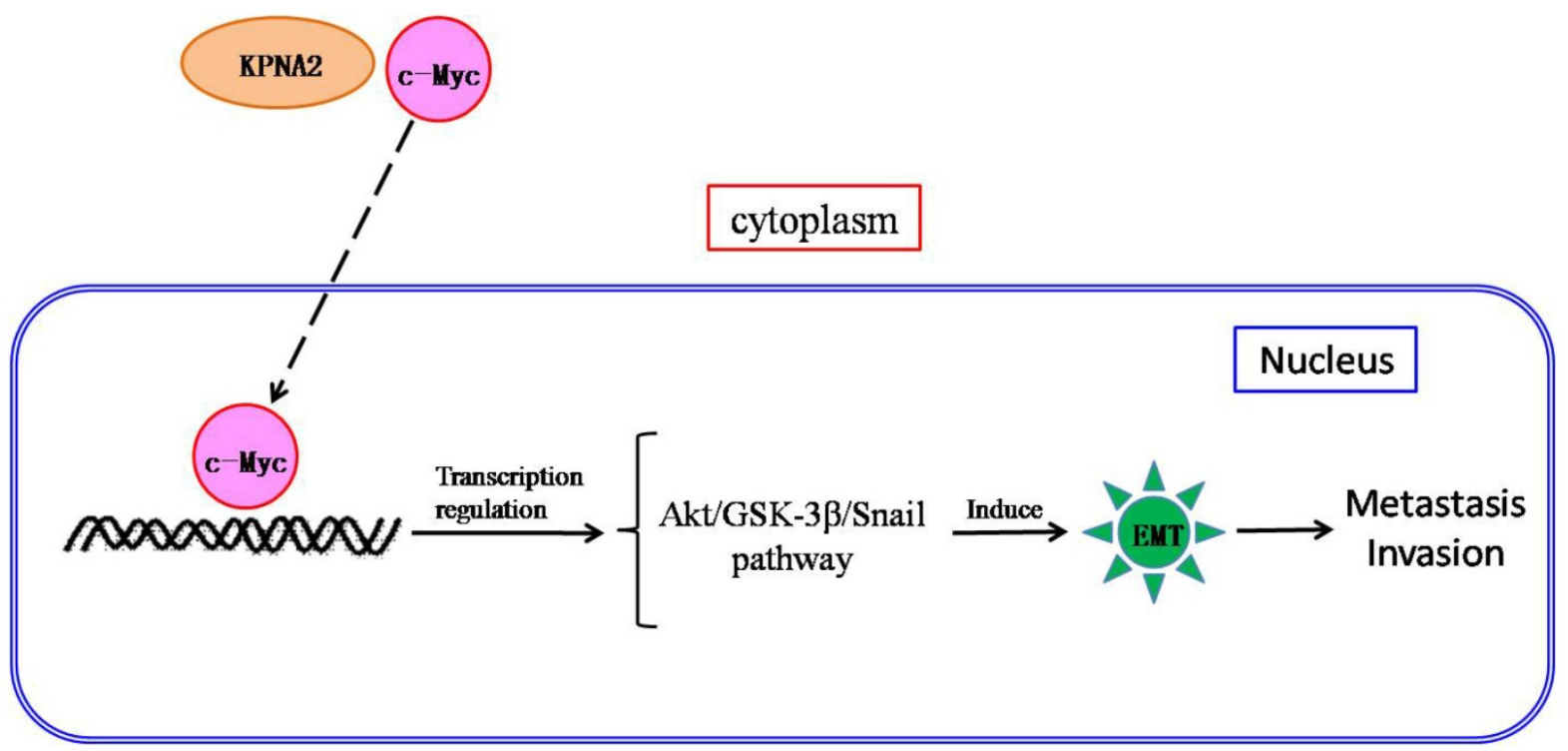

Figure 5. Scheme of the c-Myc-mediated KPNA2-induced epithelial-to-mesenchymal transition (EMT) in EOC cells. KPNA2 induces EMT, which subsequently contributes to invasion by EOC cells. In addition, KPNA2 also transports c-Myc into the nucleus and triggers the Akt/GSK-3ß/Snail pathway, which is required for KPNA2-induced EMT.

\section{Discussion}

KPNA2 mediates signaling factor import into the nucleus, and export of response molecules to the cytoplasm [12]. KPNA2 is suggested to be involved in a variety of malignancies, but the mechanism remains uncertain $[2,13]$. Previously, we showed that that mir-26b suppresses EOC cell growth and metastasis by decreasing KPNA2 and octamer-binding transcription factor 4 expression [14]. The key outcome of the present work is that migration and invasion of EOC cells were significantly increased by KPNA2 overexpression. Such overexpression also resulted in an increase in the EMT characteristics of EOC cells: epithelial cell markers were decreased and mesenchymal markers were increased. EMT is important for invasion, metastasis and drug-resistance of cancer cells [15].

EMT is regulated by various signaling pathways, including nuclear factor kappa $\mathrm{B}$, Wnt, transforming growth factor- $\beta$, Notch, and Akt/GSK-3 $\beta /$ Snail [15]. Overexpression of KPNA2 increased the expression of phosphorylated Akt and GSK-3 $\beta$, whereas knockdown of KPNA2 decreased them. The latter effect was probably mediated by inhibition of c-Myc transactivity and Akt kinase activity, and suppression of FOXO3a activity [16]. FOXO3a is a transcription factor whose activity is principally regulated by post-translational modification (PTM) and nuclear-cytoplasmic shuttling; PI3K/AKT pathway-regulated phosphorylation is the principal PTM that controls the subcellular localization of FOXO3a $[17,18]$. In addition, Spalt Like Transcription
Factor 4, which is an EMT and drug-resistance inducer, was shown to induce EMT by regulating c-Myc [19], and activated FOXO3a played a key role in doxorubicin-induced EMT in hepatocellular carcinoma cells [20]. The findings of the present study strongly suggest that KPNA2 functions as an oncogenic protein in the development and progression of EOC. Downregulation of KPNA2 significantly decreases the invasiveness of EOC cells and reverses EMT by inhibiting the Akt/GSK-3 $\beta /$ Snail pathway.

We propose a mechanism for the role of KPNA2 in EOC pathogenesis: KPNA2 may transport c-Myc into the nucleus where it regulates transactivity, triggering Akt/GSK-3 $\beta /$ Snail pathway-mediated EMT and subsequent metastasis (Fig. 5). Further research is underway to validate both this hypothesis and KPNA2 as a therapeutic target for EOC.

\section{Acknowledgement}

This work was supported by the National Natural Science Foundation of China [grant numbers 81460393 to Long Huang, 81372275 to Min Zheng].

\section{Competing Interests}

The authors have declared that no competing interest exists.

\section{References}

1. Siegel R, Ma J, Zou Z, et al. Cancer statistics, 2014. CA Cancer J Clin 2014;64:9-29.

2. Winnepenninckx V, Lazar V, Michiels S, et al. Gene expression profiling of primary cutaneous melanoma and clinical outcome. J Natl Cancer Inst 2006;98: $472-482$. 
3. Watt PJ, Maske CP, Hendricks DT, et al. The karyopherin proteins, Crm 1 and karyopherinbeta1, are overexpressed in cervical cancer and are critical for cancer cell survival and proliferation. Int J Cancer 2009; 124: 1829-1840.

4. Sakai M, Sohda M, Miyazaki T, et al. Significance of karyopherin-\{alpha\} 2 (KPNA2) expression in esophageal squamous cell carcinoma. Anticancer Res 2010; 30: 851-856.

5. Wang CI, Wang CL, Wang CW, et al. Importin subunit alpha-2 is identified as a potential biomarker for non-small cell lung cancer by integration of the cancer cell secretome and tissue transcriptome. Int J Cancer 2011; 128: 2364-2372.

6. Mortezavi A, Hermanns $\mathrm{T}$, Seifert $\mathrm{HH}$, et al. KPNA2 expression is an independent adverse predictor of biochemical recurrence after radical prostatectomy. Clin Cancer Res 2011;17: 1111-1121.

7. Gousias K, Becker AJ, Simon M, et al. Nuclear karyopherin a2: a novel biomarker for infiltrative astrocytomas. J Neurooncol 2012;109: 545-553.

8. Yoshitake K, Tanaka S, Mogushi K, et al. Importin-alpha1 as a novel prognostic target for hepatocellular carcinoma. Ann Surg Oncol 2011;18: 2093-2103.

9. Jensen JB, Munksgaard PP, Sorensen CM, et al. High expression of karyopherin-alpha2 defines poor prognosis in non-muscle invasive bladder cancer and in patients within vasive bladder cance rundergoing radicalcystectomy. Eur Urol 2011;59: 841-848.

10. Zheng M, Tang L, Huang L, Ding H, Liao WT, Zeng MS, Wang HY: Overexpression of karyopherin-2 in epithelial ovarian cancer and correlation with poor prognosis. Obstet Gynecol 2010, 116(4): 884-891.

11. Li J, Zhang N, Song LB, et al. Astrocyte elevated gene-1 is a novel prognostic maker for breast cancer progression and overall patient survival. Clin cancer Res 2008;14: 3319-3326.

12. Poon IK, Jans DA. Regulation of nuclear transport: central role in development and transformation. Traffic 2005; 6: 173-186.

13. Sakai M, Sohda M, Miyazaki T, et al. Significance of karyopherin-\{alpha\} 2 (KPNA2) expression in esophageal squamous cell carcinoma. Anticancer Res 2010; 30: 851-856.

14. Lin JX, Zhang L, Huang $\mathrm{H}$, et al. Mir-26b suppresses epithelial ovarian carcinoma cell growth and metastasis through decreasing the expression of KPNA2 and OCT4. Oncotarget 2015; ISSN:1949-2553.

15. Polyak K, Weinberg RA. Transitions between epithelial and mesenchymal states: acquisition of malignant and stem cell traits. Nat Rev Cancer 2009;9: 265-273.

16. Huang L, Wang HY, Li JD, et al. KPNA2 promotes cell proliferation and tumorigenicity in epithelial ovarian carcinoma through upregulation of c-Myc and downregulation of FOXO3a. Cell Death Dis 2013; 4:e745.

17. Trotman LC, Alimonti A, Scaglioni PP, et al. Identification of a tumour suppressor network opposing nuclear Akt function. Nature 2006; 441: 523-527.

18. Hu MC, Lee DF, Xia W, et al. IkappaB kinase promotes tumorigenesis through inhibition of forkhead FOXO3a. Cell 2004; 117: 225-237.

19. Liu L, Zhang J, Yang X, et al. SALL4 as an Epithelial Mesenchymal Transition and Drug Resistance Inducer through the Regulation of c-Myc in Endometrial Cancer. PLoS One 2015;10(9):e0138515.

20. Zhou Y, Liang C, Xue F, et al. Salinomycin decreases doxorubicin resistance in hepatocellular carcinoma cells by inhibiting the $\beta$-catenin/TCF complex association via FOXO3a activation. Oncotarget 2015; 6(12):10350-65. 